\title{
A Fast Region-Based Segmentation Model with Gaussian Kernel of Fractional Order
}

\author{
Bo Chen, Qing-Hua Zou, Wen-Sheng Chen, and Yan Li \\ College of Mathematics and Computational Science, Shenzhen University, Shenzhen 518060, China \\ Correspondence should be addressed to Wen-Sheng Chen; chenws@szu.edu.cn
}

Received 11 August 2013; Revised 3 September 2013; Accepted 3 September 2013

Academic Editor: Ming Li

Copyright (C) 2013 Bo Chen et al. This is an open access article distributed under the Creative Commons Attribution License, which permits unrestricted use, distribution, and reproduction in any medium, provided the original work is properly cited.

By summarizing some classical active contour models from the view of level set representation, a simple energy function expression with the Gaussian kernel of fractional order is proposed, and then a novel region-based geometric active contour model is established. In this proposed model, the energy function with value of $[-1,1]$ is built, the local mean and global mean of the inside and outside of the evolution curve are employed, and the segmentation results are obtained by controlling the expansion and contraction of the evolution curve. The model is simple and easy to implement; it can also protect weak edges because of considering more statistical information. Experimental results on synthetic and natural images show that the proposed model is much more effective in dealing with the images with weak or blurred edges, and it takes less time.

\section{Introduction}

Image segmentation is a basic and important topic in the fields of image processing. Accurate image segmentation can provide more important information for the follow-up application, such as machine vision and motion tracking. However, segmental results are always affected by low contrast and the problems of intensity inhomogeneity. The main idea of image segmentation is to extract the concerned regions and their contours from the whole image. There have been thousands of image segmentation algorithms proposed in recent decades. Some researchers put forward the edge detection based on the gradient, derivatives, or Canny edge detection, and so on. Edge detection is good for simple image but not suitable for the clutter target boundary extraction. The main reasons are as follows. Firstly, edge extracted for complex image is often not corresponding to the target boundary. Secondly, the extracted edge is discontinuous, but the goal often needs closed boundary to separate the object from the whole image. In addition, edge detection is dependent on the local information near pixel; it has advantages sometimes, but in many cases overall appearance of the target is the key, so the concepts of the image segmentation and edge detection are not one and the same.
Regional growth is a simple technique to provide segmental region; the algorithm begins with some seed points and found pixels near the seed which has similar image characteristics, such as gray scale and color characteristics. This algorithm has been applied to Mumford-Shah function [1]. Another region-based method is active contour (AC) model [2]. Active contour model is $2 \mathrm{D}$ or $3 \mathrm{D}$ surface contour description, which involves the contour evolution under an appropriate energy in order to get a satisfactory segmentation result, such as the target boundary with the closed contour. Over the past decade, researchers have proposed many different active contour models, which are mainly divided into two categories, namely, parametric active contour models and geometric active contour models. In parametric active contour models, the parameter equation of the curve is $C(p)=$ $[x(p), y(p)]$, in which $0 \leq p \leq 1$. The parametric active contour model essentially depends on the energy function rather than the geometric figures of the contour. Therefore, this model cannot handle topology changes when it detects multiple targets, but geometric active contour model can deal with topological changes, because it uses the structure of level set, in which the curve $C$ is zero level set function $\phi(x, t)$ : $R^{n} \times[0, \infty) \rightarrow R, n=\{2,3\}$; for example, $C=\left\{x \in R^{n}:\right.$ $\phi(x, t)=0\}$. The first type of geometric active contour model 
is introduced by Caselles et al. [3]; its main idea is to use curvature and normal direction forcing curve movement, so that it stops on the edge with the edge function $g(x)=g\left(|\nabla I|^{2}\right)$, where $\nabla I$ means the gradient of the given image $I$, which has a property; that, it equals zero on the border, and others equal one. For example, $g(x)=e^{-\left(1 / \sigma_{e}^{2}\right)}\left|\nabla G_{\sigma} * I(x)\right|^{2}$, where $\sigma_{e}$ is a scale factor, $G_{\sigma}=(1 / \sqrt{2 \pi} \sigma) e^{-\left((x-\mu)^{2} / \sigma^{2}\right)}$ is a Gaussian kernel, in which $\sigma$ denotes the standard deviation of the given image, and $\mu$ denotes the expectation of the given image. Another type of geometric active contour model is the Geodesic active contour model [4], which can search for the minimum length of the edge weights under the energy function. This model is similar to the former geometric model, but there is a big difference, a vector filed term is employed in Geodesic active contour model to stop the motion curve on the weak edges. Paragios et al. put forward the famous gradient vector flow (GVF) instead of $\nabla g$ to increase the range of results, called GVF geometric AC [5]. Chan and Vese [6] proposed a new model CV AC, and Li et al. [7] proposed a new model (LBF) which uses energy function to overcome the problem of nonhomogeneity. LBF model can deal with the image of different gray levels by adding the kernel function, and it can employ local gray level information effectively.

Many structures of different level set evolution models have been summarized before. The level set evolution of the above energy functions [8] can be represented as

$$
\frac{\partial \phi}{\partial t}=\alpha_{k} K|\nabla \phi|+V_{N}|\nabla \phi|+\vec{N} \cdot \nabla \phi
$$

where $K$ is the Euclidean curvature and $\alpha_{k}, V_{N}$, and $\vec{N}$ are three parameters, which decide the speed and direction of the evolution. The term based on curvature vector is used to smooth the curve. The normal direction is used to control shrinkage and expansion of the curve and force the curve to move along the direction vector. Details are shown in Table 1 , where $\eta, \mu, \lambda_{1}$, and $\lambda_{2}$ are constant, $[\widehat{u}, \widehat{v}]$ is the GVF, and $k(x)$ is a function based on the normal curvature and GVF [9]. At the same time, $I(x)$ in Table 1 is original gray level image, $C_{\mathrm{in}}$ and $C_{\text {out }}$ are average gray values of $I(x)$ inside and outside of curve, and $e_{1}$ and $e_{2}$ are the weighted average gray values of $I(x)$ inside and outside of curve in the Gaussian window.

The rest of the paper is organized as follows: in the next two sections, we will review classical existing geometric models, Chan-Vese model and LBF model. The new model is introduced in Section 4. Some experimental results are shown in Section 5. We conclude the paper in Section 6.

\section{Chan-Vese Model}

In Chan-Vese (CV) model, we considered the simplest type of segmentation, which divided the image into the target and the background, and the distributions of the gray values of target and background are approximately constant values. CV model is based on the evolution of the level set and can deal with curve topology changes better for the curve which is
TABLE 1: The analysis table of energy function model.

\begin{tabular}{lccc}
\hline Model & $\alpha_{k}$ & $V_{N}$ & $\vec{N}$ \\
\hline Geometric AC [3] $g(x)$ & $r g(x)$ & $\overrightarrow{0}$ \\
Geodesic AC [4] & $g(x)$ & $r g(x)$ & $\nabla g(x)$ \\
GVF Geo. AC [5] & $g(x)$ & $r k(x) g(x)$ & $g(x)(1-|k(x)|[\widehat{u}, \widehat{v}])$ \\
CV AC [6] & $\eta$ & $\mu+\left(I-c_{\text {in }}\right)^{2}$ & $\overrightarrow{0}$ \\
LBF AC [7] & $\eta$ & $-\left(I-c_{\text {out }}\right)^{2}$ & $\overrightarrow{0}$ \\
\hline
\end{tabular}

expressed by the level set function. The energy function of CV model is

$$
\begin{aligned}
E\left(C, c_{1}, c_{2}\right)= & \mu_{0} \text { Length }(C) \\
& +\lambda_{1} \int_{\text {inside }(C)}\left|u_{0}(x, y)-c_{1}\right|^{2} d x d y \\
& +\lambda_{2} \int_{\text {outside }(C)}\left|u_{0}(x, y)-c_{2}\right|^{2} d x d y
\end{aligned}
$$

where $u_{0}$ is the given image, $C$ is the evolution curve, $C(s)$ : $[0,1] \rightarrow R$ is a parameter evolution curve, and $\mu_{0}$ is the weight coefficient. In the energy function, the first item is the length of the curve evolution and it can regularize the curve. The last two items are global binary fitting items. The basic idea of the model is to minimize the fitting item $F_{1}+F_{2}$, where

$$
\begin{aligned}
& F_{1}=\int_{\text {inside }(C)}\left|u_{0}-c_{1}\right|^{2} d x d y, \\
& F_{2}=\int_{\text {outside }(C)}\left|u_{0}-c_{2}\right|^{2} d x d y .
\end{aligned}
$$

The level set function $\phi$ is defined as

$$
\begin{gathered}
\phi(x, y)>0, \quad(x, y) \in \text { in }(C), \\
\phi(x, y)<0, \quad(x, y) \in \text { out }(C), \\
\phi(x, y)=0, \quad(x, y) \in(C) .
\end{gathered}
$$

The following are Dirac function and Heaviside function, respectively:

$$
\begin{gathered}
\delta(\phi)=\frac{d H(\phi)}{d \phi}, \\
H(\phi)= \begin{cases}1, & \phi \geq 0, \\
0, & \phi<0 .\end{cases}
\end{gathered}
$$

Because function $H(\phi)$ cannot directly take the derivative of $\phi$, we can replace $H$ with $H_{\varepsilon}(\phi)$ in the CV model, where

$$
H_{\varepsilon}(\phi)=\frac{1}{2}\left(1+\frac{2}{\pi} \arctan \left(\frac{\phi}{\varepsilon}\right)\right) .
$$


The level set function of the CV model is:

$$
\begin{aligned}
E\left(\phi, c_{1}, c_{2}\right)= & \mu \int_{\Omega} \delta_{\varepsilon}(\phi)|\nabla \phi| d x d y \\
& +\lambda_{1} \int_{\Omega}\left|u_{0}(x, y)-c_{1}\right|^{2} H_{\varepsilon}(\phi) d x d y \\
& +\lambda_{2} \int_{\Omega}\left|u_{0}(x, y)-c_{2}\right|^{2}\left(1-H_{\varepsilon}(\phi)\right) d x d y,
\end{aligned}
$$

where

$$
\delta_{\varepsilon}(\phi)=H_{\varepsilon}^{\prime}(\phi)=\frac{1}{\pi} \frac{\varepsilon}{\varepsilon^{2}+\pi^{2}} .
$$

From the definition of the level set function $\phi$, expressions of $c_{1}, c_{2}$ are, respectively, as follows:

$$
\begin{gathered}
c_{1}=\frac{\int_{\Omega} u_{0}(x, y) H(\phi(x, y)) d x d y}{\int_{\Omega} H(\phi(x, y)) d x d y}, \\
c_{2}=\frac{\int_{\Omega} u_{0}(x, y)(1-H(\phi(x, y))) d x d y}{\int_{\Omega}(1-H(\phi(x, y))) d x d y} .
\end{gathered}
$$

According to Euler-Lagrange equation, the level set expression of CV model is obtained as

$$
\frac{\partial \phi}{\partial t}=\delta(\phi)\left[\mu \operatorname{div}\left(\frac{\nabla \phi}{|\nabla \phi|}\right)-\lambda_{1}\left(u_{0}-c_{1}\right)^{2}+\lambda_{2}\left(u_{0}-c_{2}\right)^{2}\right] .
$$

\section{LBF Model}

LBF model defines a local binary fitting energy item, which is actually a kernel function; the model is as follows:

$$
\begin{aligned}
& E\left(C, f_{1}, f_{2}\right) \\
& =\lambda_{1} \int_{\Omega} \int_{\text {inside }(C)} K_{\sigma}(x-y)\left|I(y)-f_{1}(x)\right|^{2} d y d x \\
& \quad+\lambda_{2} \int_{\Omega} \int_{\text {outside }(C)} K_{\sigma}(x-y)\left|I(y)-f_{2}(x)\right|^{2} d y d x,
\end{aligned}
$$

where $I: \Omega \rightarrow R$ is the original image, $(x, y) \in \Omega, K_{\sigma}=$ $\left(1 / 2 \pi \sigma^{2}\right) e^{-\left(\left(x-\mu_{x}\right)^{2}+\left(y-\mu_{y}\right)^{2} / \sigma^{2}\right)}$ and is a Gaussian kernel function, $\mu_{x}$ and $\mu_{y}$ are its expectancies, and $\sigma$ is its standard deviation. $f_{1}$ and $f_{2}$ are the image fitting function of the local gray level inside and outside of the contour.

The variational level set function of (11) which is got by Euler-Lagrange equation is as follows:

$$
\frac{\partial \phi}{\partial t}=-\delta(\phi)\left(\lambda_{1} e_{1}-\lambda_{2} e_{2}\right)
$$

By introducing a sign distance constraint and length constraint item, the level set evolution equation is

$$
\begin{aligned}
\frac{\partial \phi}{\partial t}= & \mu\left(\Delta \phi-\operatorname{div}\left(\frac{\nabla \phi}{|\nabla \phi|}\right)\right)+\nu \delta(\phi) \operatorname{div}\left(\frac{\nabla \phi}{|\nabla \phi|}\right) \\
& -\delta(\phi)\left(\lambda_{1} e_{1}-\lambda_{2} e_{2}\right),
\end{aligned}
$$

where functions $e_{1}, e_{2}$ and $f_{1}, f_{2}$ are as follows, respectively:

$$
\begin{gathered}
e_{1}(x)=\int_{\Omega} K_{\sigma}(y-x)\left|I(x)-f_{1}(y)\right|^{2} d y, \\
e_{2}(x)=\int_{\Omega} K_{\sigma}(y-x)\left|I(x)-f_{2}(y)\right|^{2} d y, \\
f_{1}=\frac{K_{\sigma} *[H(\phi) I(x)]}{K_{\sigma} * H(\phi)}, \\
f_{2}=\frac{K_{\sigma} *[(1-H(\phi)) I(x)]}{K_{\sigma} *((1-H(\phi)))} .
\end{gathered}
$$

Equation (15) shows that $f_{1}$ and $f_{2}$ are the weighted average gray values with the Gaussian window inside and outside of contour. Obviously, they share the local characteristics, so that the segmentation of original image by LBF model is more accurate.

\section{Proposed Region-Based Model with Gaussian Kernel of Fractional Order}

In order to get better image segmentation results effectively and construct a fast region-based segmentation model, we should keep the energy functional as simple as possible, and energy information must be used effectively. Based on the law of some classical energy function expressions of active contour models summarized in Table 1, we know that the term $V_{N}$ in energy function is very important, and many models made a breakthrough on it. At the same time, the item $\vec{N}$ of the models is often set as $\overrightarrow{0}$, and item $\alpha_{k}$ is only ordinary parameters. Therefore, only keeping item $V_{N}$ in the new model will simplify the expression of energy function.

In order to avoid jumping internally, level set function initialized by symbolic distance function (SDF) in the traditional level set method, but it usually needs to be reinitialized. This will lead to the fact that it is hard to decide when to reinitialize and how to reinitialize, as it is hard to find boundary when the zero level set is away from the inner region. So reinitialization is a very complex operation problem. To solve this problem, we propose a new level set method. At the same time, fractional systems $[10,11]$ gain increasing attention in applied sciences, and functions of fractional order are more flexible, so the new method uses a Gaussian filter with fractional order to regularize binary level set function. The traditional level set method uses curvature item $\operatorname{div}(\nabla \phi /|\nabla \phi|)|\nabla \phi|$ to regularize the level set function, and letting $|\nabla \phi|=1$ [12], it can replace the regular items with Laplacian. Based on scale space theory in [13], a function with the Laplacian evolution is equivalent to using a Gaussian filter. Then, we filter the initial conditions of the level set function with Gaussian kernel filters of the level set function, and $\sigma$ controls the regular strength, similar to the item $\eta$ in Table 1 . With Gaussian kernel function, the item $\operatorname{div}(\nabla \phi /|\nabla \phi|)|\nabla \phi|$ (similar to the item $\alpha_{k}$ in Table 1) can be removed, so the key of whole model is the choice of item $V_{N}$.

From the view of level set function, we need to find a function that can adjust the pressure inside and outside of the interest areas. It drives the curve to contract when the curve 


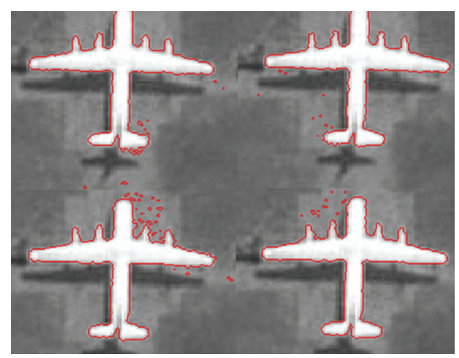

(a)

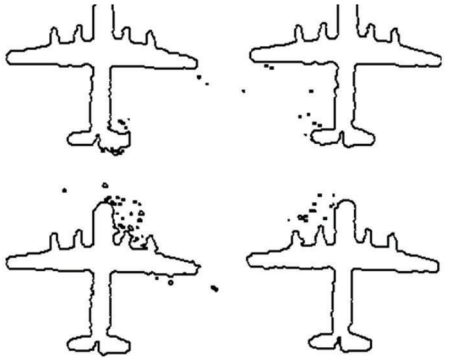

(d)

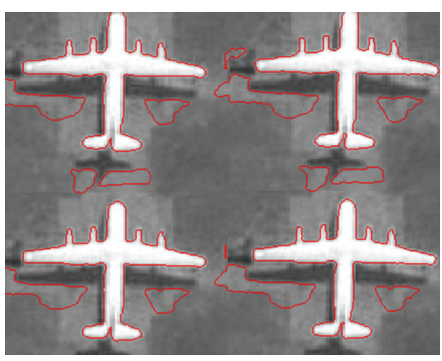

(b)

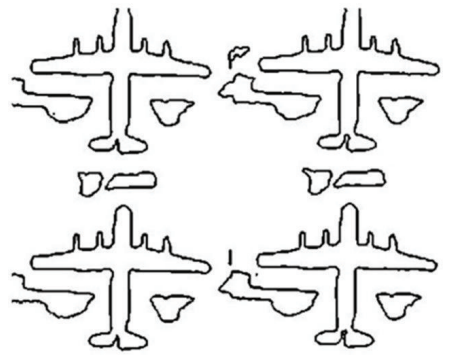

(e)

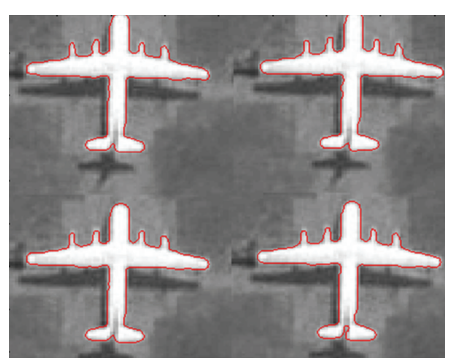

(c)
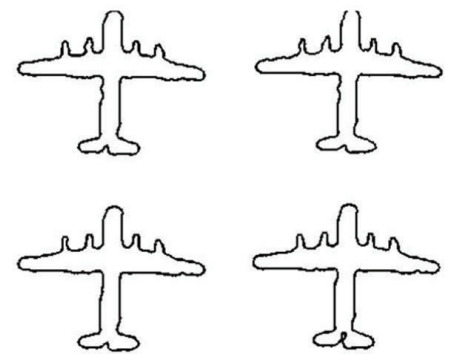

(f)

FIGURE 1: The segmentation results of the aircraft composite image: (a) and (d) results using CV model, (b) and (e) results using LBF model, and (c) and (f) results using the new model.

is outside the target and expands when the curve is within the target. Based on SPF function with value of $[-1,1]$ defined in [14], we can construct a function as follow:

$$
f(I(x))=\frac{\left(c_{1}+c_{2}+f_{1}+f_{2}\right) / 4-I(x)}{\max \left(\left|\left(c_{1}+c_{2}+f_{1}+f_{2}\right) / 4-I(x)\right|\right)}, \quad x \in \Omega,
$$

where

$$
\begin{gathered}
\operatorname{Min}(I(x)) \leq c_{1}, c_{2}, f_{1}, f_{2} \leq \operatorname{Max}(I(x)), \\
\operatorname{Min}(I(x)) \leq \frac{\left(c_{1}+c_{2}+f_{1}+f_{2}\right)}{4} \leq \operatorname{Max}(I(x)), \\
f_{1}=\frac{K_{\sigma} *[H(\phi) I(x)]}{K_{\sigma} * H(\phi)}, \\
f_{2}=\frac{K_{\sigma} *[(1-H(\phi)) I(x)]}{K_{\sigma} *(1-H(\phi))}, \\
K_{\sigma}(\alpha)=\frac{1}{2 \pi \sigma^{2}} e^{-\left(\|x-\mu\|^{\alpha} / \sigma^{2}\right)},
\end{gathered}
$$

where $K_{\sigma}(\alpha)$ is the Gaussian kernel of fractional order $\alpha$. It takes the ordinary Gaussian kernel as its special case when $\alpha=2$. Thus, it is more flexible than the ordinary one. We must emphasize that the parameter $\alpha$ may be different from fractal parameters $[15,16]$. In this paper, we call $\alpha$, likely informal, the fractional order of the Gaussian kernel expressed by (19). In the following experiments, we try different values of the fractal order $\alpha$ in the evolution level set function. The value of the function is between -1 and 1 . It drives curve to contract externally the target and expands when the curve is within the target. According to the summary of the classic model of general expression (1), we only keep item $V_{N}$, so we obtain the corresponding variational level set formulation as follows:

$$
\frac{\partial \phi}{\partial t}=f(I(x))|\nabla \phi|, \quad x \in \Omega .
$$

By adding a parameter, the final level set equation of the new model is

$$
\frac{\partial \phi}{\partial t}=\eta f(I(x))|\nabla \phi|, \quad x \in \Omega .
$$

The main algorithm of the new model is as follows.

Step 1: initialize the level set function $\phi$.

Step 2: compute the mean and the weighted average values of inside and outside of the curve $c_{1}, c_{2}, f_{1}$, and $f_{2}$.

Step 3: compute the evolution of level set function by (21).

Step 4: use Gaussian filter to regularize level set function, $\phi=$ $\phi * G_{\sigma}$.

Step 5: repeat Steps 2 to 4 until convergence.

\section{Experimental Results}

In this section, we will show some experimental results of the proposed model on synthetic image and nature image; the results also will be compared with those got by the conventional CV model and LBF model. Our algorithm is implemented in Windows 7 Operating System, i3 Dual Core CPU $2.13 \mathrm{GHz}$ and $2 \mathrm{~GB}$ RAM. The initial value and parameters such as time step take different values in the specific experiments in this paper.

Figures 1(d)-1(f) got with 2000, 1500, 40 iterations separately and consume time $t=217.6 \mathrm{~s}, 232.6 \mathrm{~s}, 13.8 \mathrm{~s}$ in turn. We can see that Chan-Vese model and LBF model cannot get 


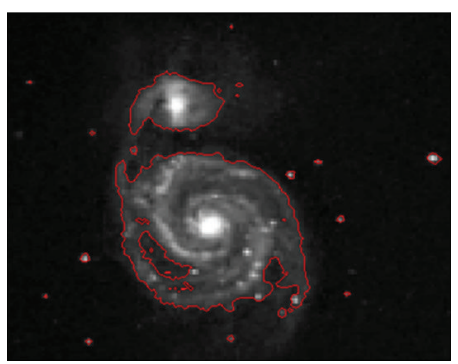

(a)

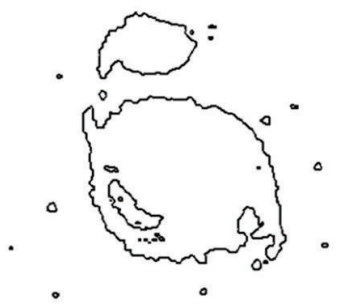

(d)

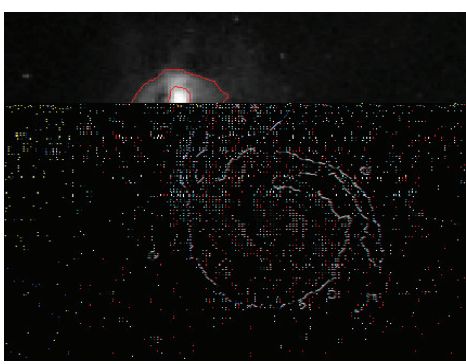

(b)

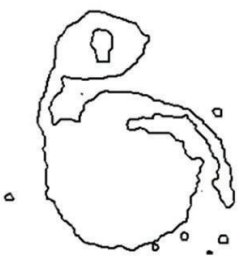

(e)

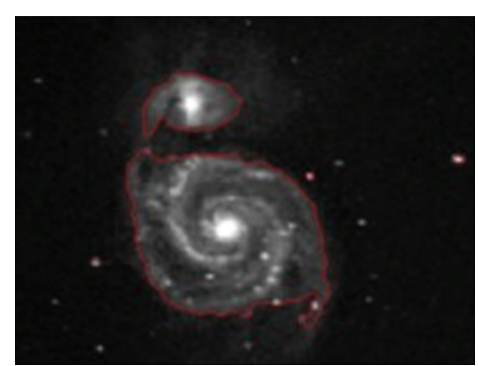

(c)

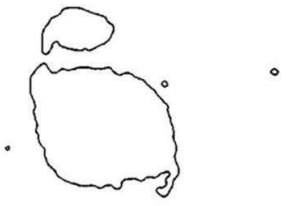

(f)

Figure 2: The segmentation results of the natural image: (a) and (d) results using CV model, (b) and (e) results using LBF model, and (c) and (f) results using the new model.

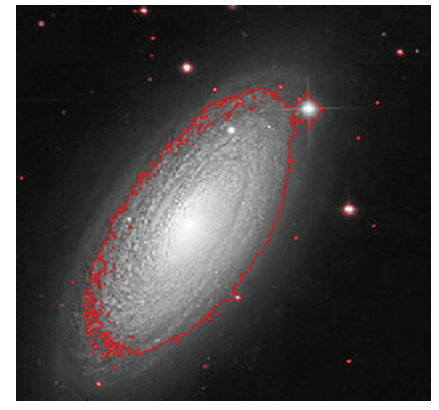

(a)

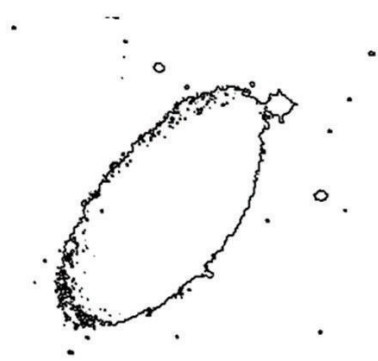

(d)

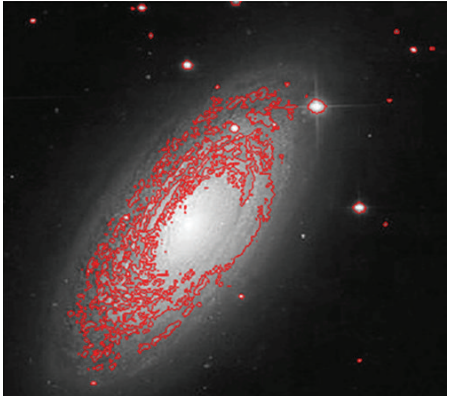

(b)

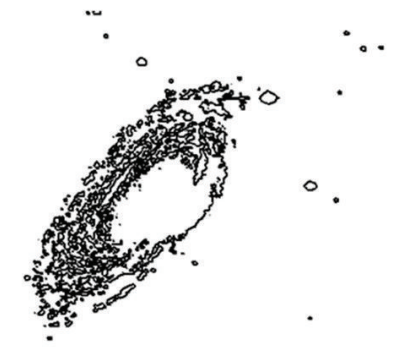

(e)

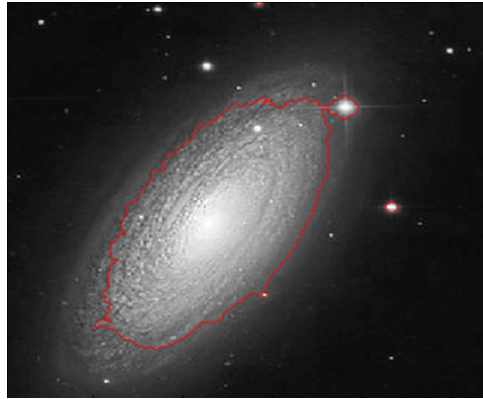

(c)

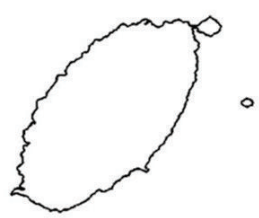

(f)

FIGURE 3: The segmentation results of the natural image: (a) and (d) results using CV model, (b) and (e) results using LBF model, and (c) and (f) results using the new model.

satisfactory result. While here the proposed model gets satisfactory result, meanwhile the proposed model costs less time.

Figure 2 shows the experimental results of natural figure with objects having inhomogeneous background. CV model, LBF model, and the proposed model share the same environment of the initial value. We can find that the gray level of the natural star figure is extremely inhomogeneous easily.
The first line shows the segmentation result of CV model. The second line shows the segmentation result of LBF model. The third line shows the segmentation result of new model. The segmentation images reveal that the proposed model gets the most ideal segmentation result.

Figure 3 shows the experimental results of natural figure with CV model, LBF model, and the proposed model; gray 


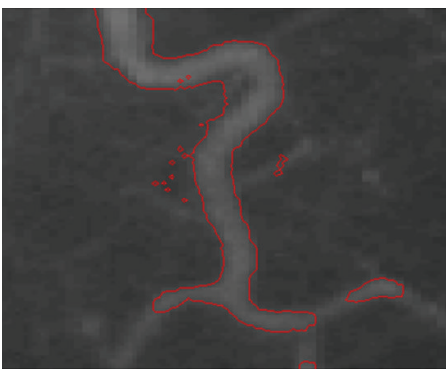

(a)

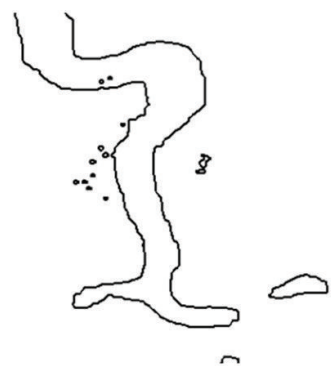

(d)

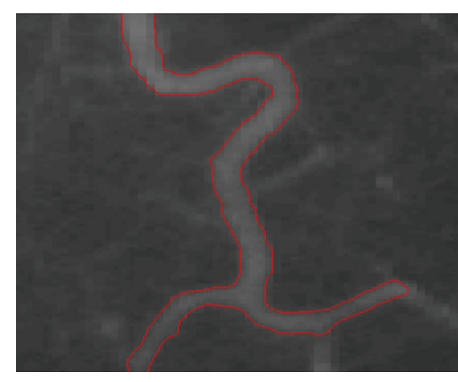

(b)

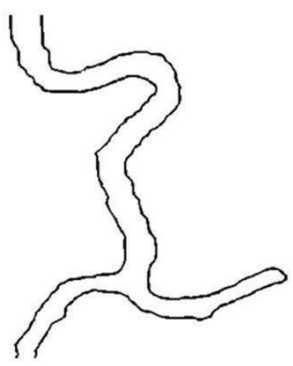

(e)

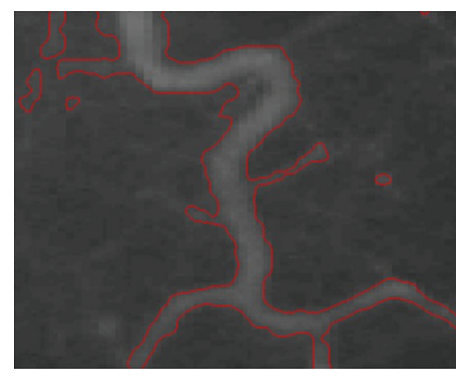

(c)

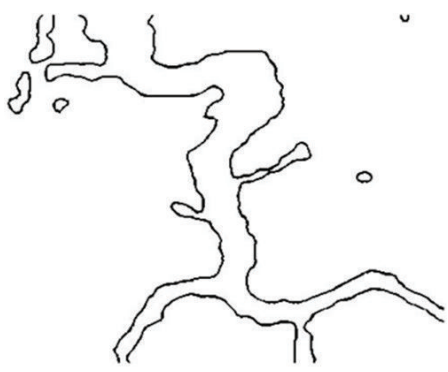

(f)

FIGURE 4: The segmentation results of the blood vessels image: (a) and (d) results using CV model, (b) and (e) results using LBF model, and (c) and (f) results using the new model.

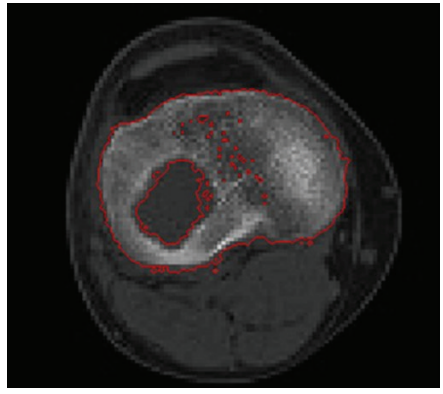

(a)

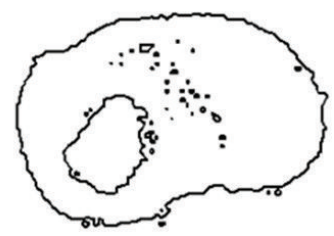

(d)

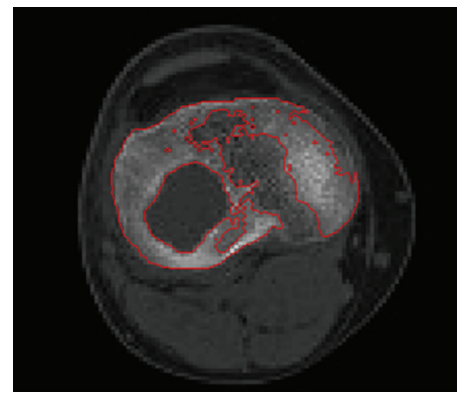

(b)

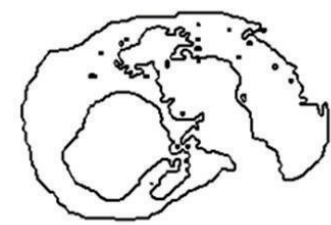

(e)

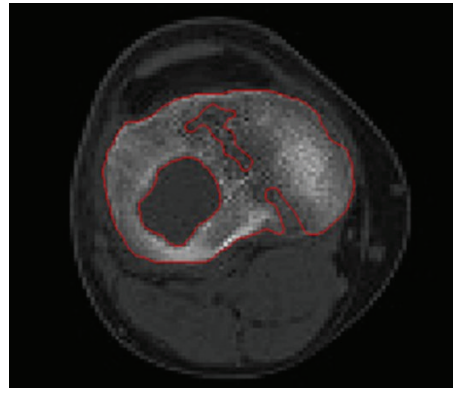

(c)

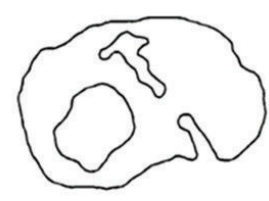

(f)

FIGURE 5: The segmentation results of the CT image: (a) and (d) results using CV model, (b) and (e) results using LBF model, and (c) and (f) results using the new model.

level of the image is extremely inhomogeneous. The three models share the same initial value. Figures $3(\mathrm{a})$ and $3(\mathrm{~d})$ show the segmentation result of CV model; Figures $3(\mathrm{~b})$ and $3(\mathrm{e})$ show the segmentation result of LBF model; Figures 3(c) and $3(\mathrm{f})$ show the segmentation result of the new model. The segmentation results show that the proposed model gets the satisfactory complete result, while the CV model and LBF model have some redundant and inaccurate segmentation.
In Figure 4, the experiment shows the segmentation results of a gray matter blood vessels image. The first column shows the segmentation results of Chan-Vese model. The second column shows the results of LBF model. The third column shows the result of proposed model. Figures $4(\mathrm{~d})-4(\mathrm{f})$ got with 1000, 5000, 40 iterations separately and consume time $t=79.2 \mathrm{~s}, 174.2 \mathrm{~s}, 6.1 \mathrm{~s}$ in turn. Because the mean information of Chan-Vese model is very sensitive to inhomogeneity 
image, it fails to extract the accurate contour. The LBF model is better than Chan-Vese model, but it gets many iterations and it is very sensitive to initial value. As shown in the low left of the segmental contour, the proposed model got a better segmentation result.

In Figure 5, experimental results show the segmentation results of CT bone images. The initialization is a single circle. The first column shows the segmentation result of ChanVese model. The second column shows the result of the LBF model. The third row shows the result of proposed model. Figures 5(d)-5(f) are the corresponding contours to Figures 5(a)-5(c). Figures 5(d)-5(f) got with 50, 1000, 80 iterations separately and consume time $t=4.0 \mathrm{~s}, 34.6 \mathrm{~s}, 12.5 \mathrm{~s}$ in turn. As seen from Figure 5, the new method can get more complete contour than Chan-Vese model and the LBF model and take less time.

\section{Conclusion}

Inspired by the idea of some classical energy function expressions of active contour model, from the view of level set representation, a novel fast region-based segmentation model with Gaussian kernel of fractional order is proposed. The model is simple and easy to be implementated, and it can protect weak edges because of considering more statistical information. The experimental results on synthetic images and natural images show that the proposed model is superior to the traditional methods. The new model is much more effective in dealing with the images with weak or blurred edges, and it takes less time.

\section{Acknowledgments}

This paper is partially supported by NSFC (61272252) and Science and Technology Planning Project of Shenzhen City (JC201105130461A, JCYJ20120613102415154, ZYC201105130115A, and JCYJ20130326111024546). The authors also would like to thank the Key Laboratory of Medical Image Processing in Southern Medical University for providing original medical images for experiment.

\section{References}

[1] D. Mumford and J. Shah, "Optimal approximations by piecewise smooth functions and associated variational problems," Communications on Pure and Applied Mathematics, vol. 42, no. 5, pp. 577-685, 1989.

[2] M. Kass, A. Witkin, and D. Terzopoulos, "Snakes: active contour models," International Journal of Computer Vision, vol. 1, no. 4, pp. 321-331, 1988.

[3] V. Caselles, F. Catté, T. Coll, and F. Dibos, "A geometric model for active contours in image processing," Numerische Mathematik, vol. 66, no. 1, pp. 1-31, 1993.

[4] V. Caselles, R. Kimmel, and G. Sapiro, "Geodesic active contours," International Journal of Computer Vision, vol. 22, no. 1, pp. 61-79, 1997.

[5] N. Paragios, O. Mellina-Gottardo, and V. Ramesh, "Gradient vector flow fast geometric active contours," IEEE Transactions on Pattern Analysis and Machine Intelligence, vol. 26, no. 3, pp. 402-407, 2004.
[6] T. F. Chan and L. A. Vese, "Active contours without edges," IEEE Transactions on Image Processing, vol. 10, no. 2, pp. 266-277, 2001.

[7] C. Li, C.-Y. Kao, J. C. Gore, and Z. Ding, "Implicit active contours driven by local binary fitting energy," in Proceedings of the IEEE Computer Society Conference on Computer Vision and Pattern Recognition (CVPR '07), Minneapolis, Minn, USA, June 2007.

[8] P. T. H. Truc, T.-S. Kim, S. Lee, and Y.-K. Lee, "Homogeneityand density distance-driven active contours for medical image segmentation," Computers in Biology and Medicine, vol. 41, no. 5, pp. 292-301, 2011.

[9] A. Vasilevskiy and K. Siddiqi, "Flux maximizing geometric flows," IEEE Transactions on Pattern Analysis and Machine Intelligence, vol. 24, no. 12, pp. 1565-1578, 2002.

[10] M. Li, "Approximating ideal filters by systems of fractional order," Computational and Mathematical Methods in Medicine, vol. 2012, Article ID 365054, 6 pages, 2012.

[11] M. Li, S. C. Lim, and S. Chen, "Exact solution of impulse response to a class of fractional oscillators and its stability," Mathematical Problems in Engineering, vol. 2011, Article ID 657839, 9 pages, 2011.

[12] S. Osher and R. Fedkiw, Level Set Methods and Dynamic Implicit Surfaces, vol. 153 of Applied Mathematical Sciences, Springer, New York, NY, USA, 2003.

[13] P. Perona and J. Malik, "Scale-space and edge detection using anisotropic diffusion," IEEE Transactions on Pattern Analysis and Machine Intelligence, vol. 12, no. 7, pp. 629-639, 1990.

[14] K. Z. Zhang, L. Zhang, H. H. Song, and W. G. Zhou, "Active contours with selective local or global segmentation: a new formulation and level set methed," Image and Vision Computing, vol. 28, no. 4, pp. 668-676, 2010.

[15] M. Li and W. Zhao, "Quantitatively investigating locally weak stationarity of modified multifractional Gaussian noise," Physica A, vol. 391, no. 24, pp. 6268-6278, 2012.

[16] M. Li and W. Zhao, "On $1 / f$ noise," Mathematical Problems in Engineering, vol. 2012, Article ID 673648, 23 pages, 2012. 


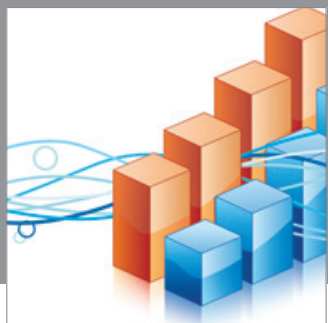

Advances in

Operations Research

mansans

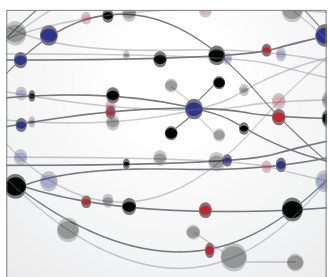

The Scientific World Journal
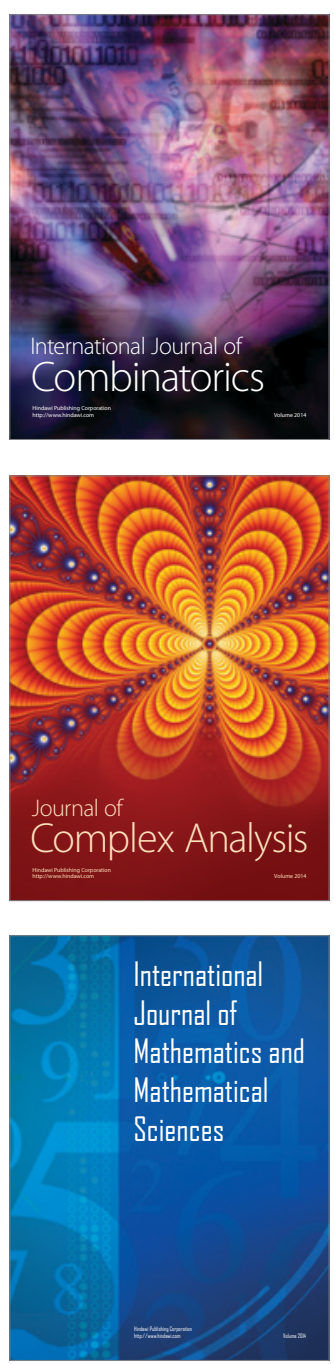
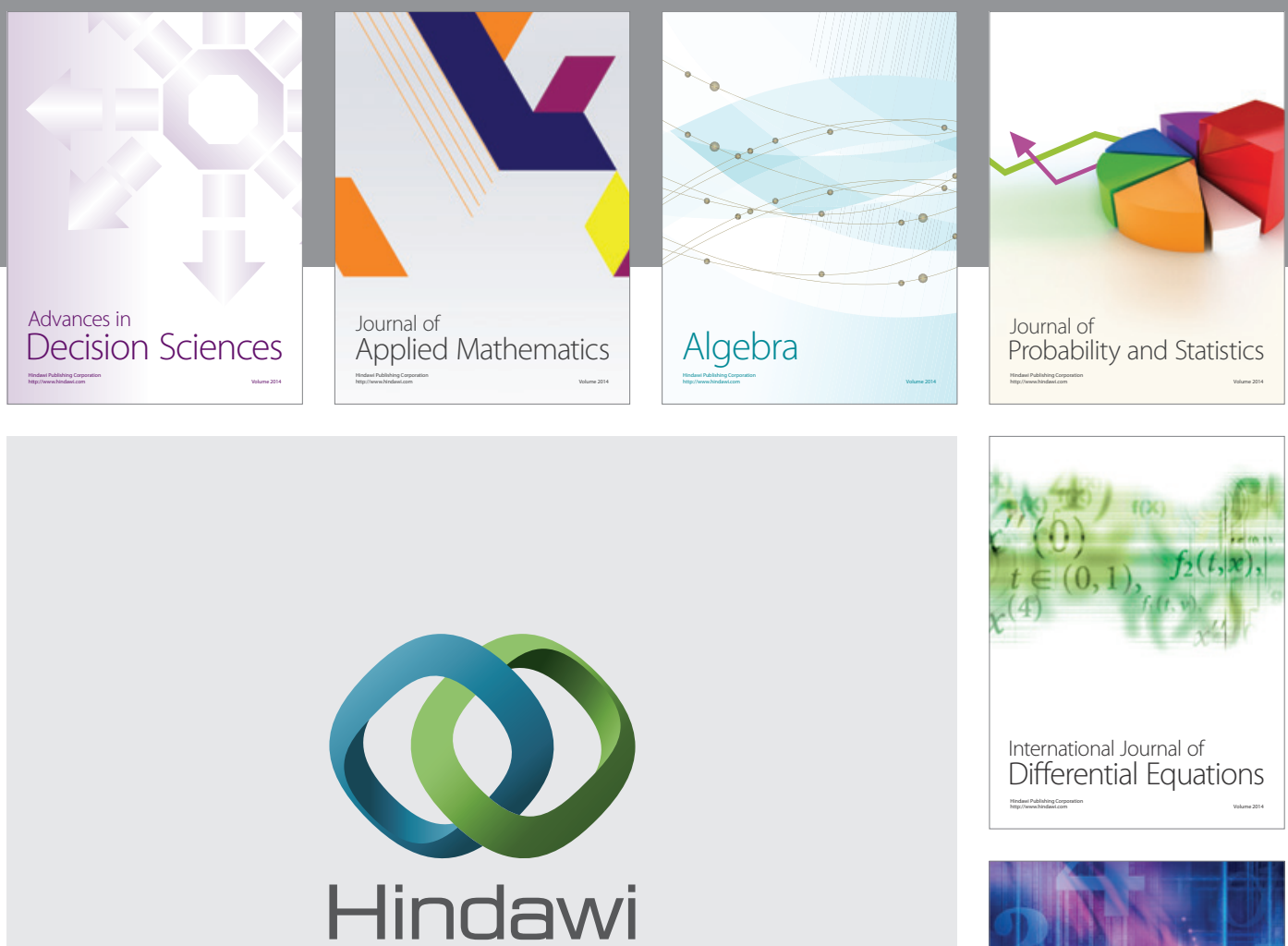

Submit your manuscripts at http://www.hindawi.com
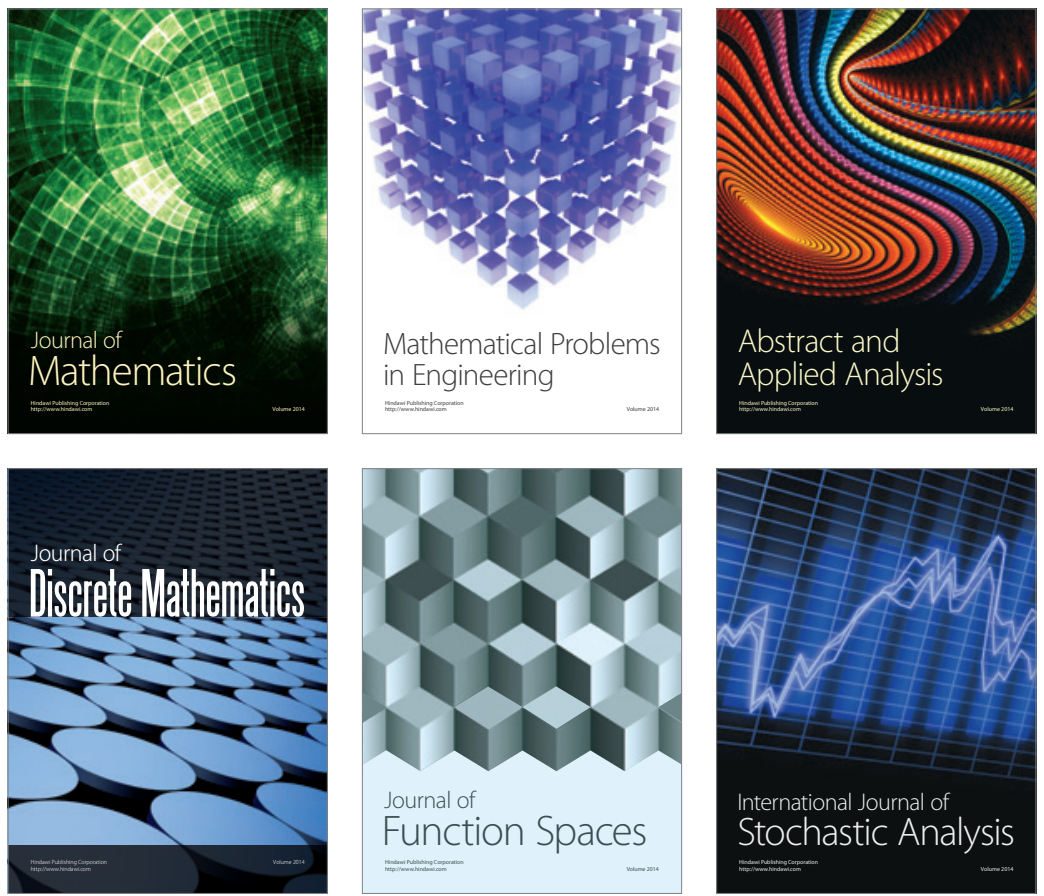

Journal of

Function Spaces

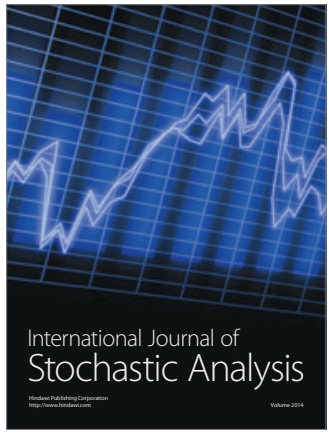

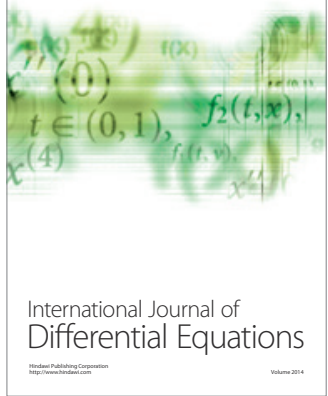
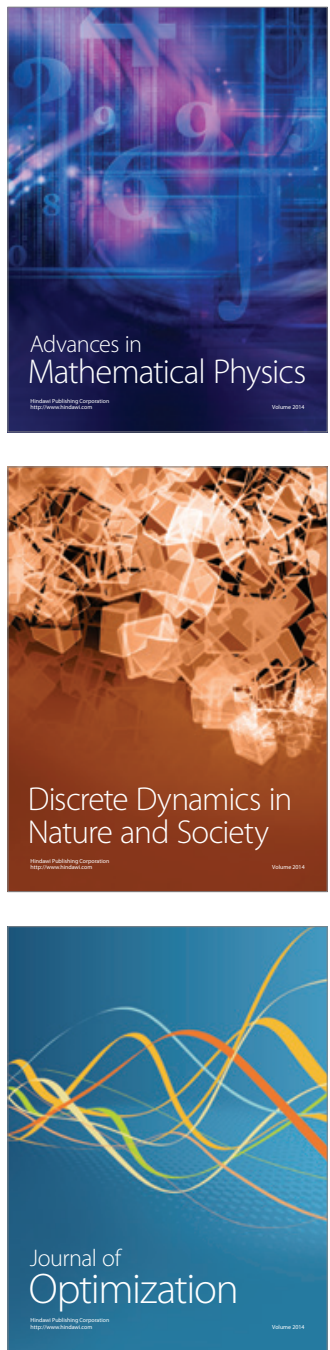